\title{
Progesterone suppresses triple-negative breast cancer growth and metastasis to the brain via membrane progesterone receptor $\alpha$
}

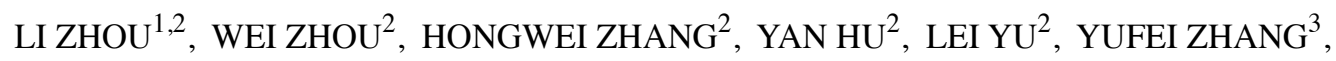 \\ YANLI ZHANG $^{2}$, SHUANG WANG $^{2}$, PENG WANG $^{2}$ and WEI XIA ${ }^{4}$ \\ ${ }^{1}$ Department of Oncology and Hematology, Shanghai Pudong New District Zhoupu Hospital, \\ Shanghai 201318; Departments of ${ }^{2}$ Oncology, ${ }^{3}$ Intervention and ${ }^{4}$ Nuclear Medicine, \\ Shanghai Seventh People's Hospital, Shanghai 200137, P.R. China
}

Received November 10, 2015; Accepted June 12, 2017

DOI: $10.3892 /$ ijmm.2017.3060

\begin{abstract}
Progesterone plays an important role in mammary epithelial cell proliferation and differentiation. Evidence from experimental and clinical studies indicates that progesterone is a risk factor for breast cancer under certain conditions through binding nuclear progesterone receptor (PR). These mechanisms, however, are not applicable to triple-negative breast cancer(TNBC) due to the lack of PR in these cancers. In this study, we demonstrate that membrane progesterone receptor $\alpha(\mathrm{mPR} \alpha)$ is expressed in TNBC tissues and the expression level of mPR $\alpha$ is negatively associated with the TNM stage. We found that progesterone suppressed the growth, migration and invasion of mPR $\alpha^{+}$human TNBC cells in vitro, which was neither mediated by PR nor by PR membrane component 1 (PGRMCl). Notably, these effects exerted by progesterone were significantly blocked by shRNA specific to $\mathrm{mPR} \alpha$. Moreover, the knockdown of $\mathrm{mPR} \alpha$ expression impaired the inhibitory effects of progesterone on $\mathrm{mPR} \alpha^{+}$tumor growth and metastasis in vivo. These data collectively indicate that progesterone suppresses TNCB growth and metastasis via $\mathrm{mPR} \alpha$, which provides evidence of the anti-neoplastic effects of progesterone-mPR $\alpha$ pathway in the treatment of human TNBC.
\end{abstract}

\section{Introduction}

Triple-negative breast cancer (TNBC), accounting for approximately $15-25 \%$ of all breast cancer cases, is characterized by the lack of estrogen receptor $\left(\mathrm{ER}^{-}\right)$, progesterone receptor $\left(\mathrm{PR}^{-}\right)$

Correspondence to: Dr Peng Wang, Shanghai Seventh People's Hospital, 358 Datong Road, Pudong, Shanghai 200137, P.R. China E-mail: 13916730828@163.com

Dr Wei Xia, Department of Nuclear Medicine, Shanghai Seventh People's Hospital, 358 Datong Road, Pudong, Shanghai 200137, P.R. China

E-mail: xiawei0910@126.com

Key words: triple-negative breast cancer, progesterone receptor, membrane progesterone receptor $\alpha$, shRNA, MB231br cells and HER2 amplification (HER2-) (1,2). Although systematic therapeutic approaches have reduced the mortality rate, TNBC is still associated with high rates of cancer recurrence, frequent metastasis to the brain and poor outcomes $(2,3)$. Therefore, an enhanced understanding of the molecular pathways involved in the progression of TNBC may be helpful in the prevention of metastasis and the design of effective therapeutic strategies for this disease.

Progesterone plays an important role in mammary epithelial cell proliferation and differentiation (4). Studies using human breast cancer cell lines and patient tumor samples, as well as clinical studies have indicated that progesterone is a risk factor for breast cancer under certain conditions (5,6; and refs therein). Classically, the effects of progesterone on cancer cells are attributed to the binding of nuclear PR, the translocation of the progesterone/PR complex into the nucleus and the subsequent activation of target genes over the course of several hours $(7,8)$. Breast cancer is a heterogeneous disease and several distinct subtypes exist, of which the triple-negative subtype has the most severe clinical prognosis $(3,9,10)$. In TNBC, these mechanisms described above are not applicable due to the lack of PR in these cancers. Thus, the role of progesterone in the pathogenesis of TNBC remains controversial, and whether progesterone is a promoter or inhibitor of TNBC has not yet been fully elucidated.

During the past decade, the discovery of membrane progesterone receptor $\alpha(\mathrm{mPR} \alpha)$, unrelated to the classical PR, in fish and its subsequent identification in mammals, suggests a potential mediator of non-traditional progestin actions $(11,12)$, particularly in tissues in which PR is absent. The broad distribution of $\mathrm{mPR} \alpha$ mRNAs in reproductive and non-reproductive tissues suggests they have diverse physiological functions in vertebrates (13-17). Recently, changes have been observed in the mRNA expression of $\mathrm{mPR} \alpha$ in malignant human breast tissues, and $\mathrm{mPR} \alpha$ has been identified as an intermediary factor of the progestin-induced intracellular signaling cascades in the PR-breast cancer cell lines in vitro $(15,18,19)$. However, the function and molecular mechanisms of action of $\mathrm{mPR} \alpha$ in mediating the effects of progesterone on TNBC cells remain unknown.

In the present study, we demonstrate that $\mathrm{mPR} \alpha$ is expressed in TNBC tissues and that the expression level of $\mathrm{mPR} \alpha$ is negatively associated the TNM stage. Progesterone suppressed the growth, migration and invasion of $m P R \alpha^{+}$human 
TNBC cells. Notably, the inhibitory effects of progesterone on $\mathrm{mPR} \alpha^{+}$human TNBC cells were not mediated by PR or by PR membrane component 1 (PGRMCl). Moreover, the knockdown of $m P R \alpha$ expression impaired the inhibitory effects of progesterone on $\mathrm{mPR} \alpha^{+}$tumor growth and metastasis in vivo. Our data collectively indicate that progesterone suppresses TNBC growth and metastasis to the brain via $m P R \alpha$, which provides evidence of the anti-neoplastic effects of the progesterone-mPR $\alpha$ pathway in the treatment of human TNBC.

\section{Materials and methods}

Cell lines and cancer tissues. Two human TNBC cell lines [MDA-MB-231 (designated as MB231) and MDA-MB-231-BR (brain-seeking cells; designated as MB231br); Type Culture Collection of the Chinese Academy of Sciences, Shanghai, China] were cultured in DMEM supplemented with $10 \%$ FBS, $100 \mathrm{U} / \mathrm{ml}$ penicillin and $100 \mu \mathrm{g} / \mathrm{ml}$ streptomycin, at $37^{\circ} \mathrm{C}$ with $5 \% \mathrm{CO}_{2}$ in a humidified incubator. Primary TNBC tissues and matched non-tumour tissues were collected from 55 patients with TNBC undergoing modified radical mastectomy between 2005-2010 at the Department of Oncology, Shanghai Seventh People's Hospital, Shanghai, China. All the tissue samples were collected after obtaining patient informed consent and ethics approval (this study was approved by the Institutional Review Board of Shanghai Seventh People's Hospital; approval no. 2015011602) and confirmed by pathological examination.

Immunohistochemistry. Briefly, the tissue sections (4- $\mu \mathrm{m}$-thick) were deparaffinized in xylene and rehydrated in graded ethanol. Following antigen retrieval, endogenous peroxidase quenching and blocking with $10 \%$ normal goat serum, the samples were incubated with primary anti-mPR $\alpha$ antibody (ab75508; Abcam, Cambridge, MA, USA) at $4^{\circ} \mathrm{C}$ overnight and then incubated with a secondary antibody (DakoCytomation, Glostrup, Denmark). The immunostained slides were counterstained with hematoxylin. In each experiment, a negative control was included in which the primary antibody was omitting. Staining was scored by a trained research pathologist who was blinded to patient clinical data.

Plasmid construction and transfection. The full-length cDNA of $\mathrm{mPR} \alpha$ was amplified using the following primers: 5'-CAT GGCGACGGTGGTGATG-3' (forward) and 5'-GGCA GCAGAAGAAATAGGCG-3' (reverse), and then subcloned into the vector, pIRES2-EGFP (BD Biosciences Clontech). The sequence for the construction of mPR $\alpha$-shRNA was sense, 5'-GGAGCTGTAAGGTCTTCTTTA-3'; and antisense, 5'-TAAAGAAGACCTTACAGCTCC-3', and then subcloned into the vector, pSUPER (GenerayBiotech Co., Ltd., Shanghai, China). Sequence fidelity and reading frame accuracy of the $\mathrm{mPR} \alpha$ expression or mPR $\alpha$-shRNA plasmid were achieved by DNA sequencing analysis. The MB231 or MB231br cells were transfected using Lipofectamine ${ }^{\circledR} 3000$ (Thermo Fisher Scientific, Inc., Waltham, MA, USA) with the mPR $\alpha$ expression plasmid or mPR $\alpha$-shRNA plasmid, respectively. The pIRES2-EGFP vector or pSUPER vector was also transfected into the MB231 or MB231br cells which served as controls. The transfected cells were then cultured in medium supplemented with G418 (Promega, Madison, WI, USA) or puromycin (InvivoGen, San Diego, CA, USA) for the establishment of stably transfected cell clones.

Reverse transcription-quantitative PCR (RT-qPCR). Total RNA was isolated using TRIzol reagent (Invitrogen Life Technologies, Carlsbad, CA, USA) and reverse transcribed using a reverse transcription kit (Promega) according to the manufacturer's instructions. Quantitative PCR (qPCR) was performed to determine the expression of $\mathrm{mPR} \alpha$ using SYBRGreen PCR master mix. The primers for $m P R \alpha$ were as follows: sense, 5'-GTGGTGATGGAGCAGATTGGT-3' and antisense, 5'-TGCCAGGAGGACGATGAATAG-3'. The primers for GAPDH were as follows: sense, 5'-TTGGCATCG TTGAGGGTCT-3' and antisense, 5'-CAGTGGGAACACGG AAAGC-3'. The relative expression ratio of $m P R \alpha$ was calculated using the $2^{-\Delta \Delta \mathrm{Cq}}$ method.

Western blot analysis. Cell lysates were prepared with RIPA (Beyotime Institute of Biotechnology, Haimen, China) and separated by SDS-polyacrylamide gel electrophoresis and transferred onto polyvinylidene fluoride membranes. Following incubation with blocking buffer (TBS + 0.1\% Tween-20 + 5\% not-fat milk), the membranes were probed with the primary antibody (anti-mPR $\alpha$ antibody; ab75508; Abcam) overnight at $4^{\circ} \mathrm{C}$. The membranes were then washed with TBST and incubated with an HRP-conjugated secondary antibody (horseradish peroxidase-labeled goat anti-rabbit IgG; A0208; Beyotime Institute of Biotechnology), and signals were detected using ECL reagent (Millipore, Billerica, MA, USA). mPR $\alpha$, caspase-3, cleaved caspase-3 and GAPDH antibodies were from Abcam.

Cell proliferation assay. Cell proliferation was determined using the Cell Counting Kit-8 (CCK-8) assay (Dojindo Molecular Technologies, Inc.). Briefly, $5 \times 10^{5}$ cells were seeded in 24-well plates and treated with various concentration of progesterone $(20 \mathrm{ng} / \mathrm{ml}, 40 \mathrm{ng} / \mathrm{ml}$ and $80 \mathrm{ng} / \mathrm{ml}$; Sigma-Aldrich, St. Louis, MO, USA), RU486 (mifepristone; EMD Chemicals, Gibbstown, NJ, USA) or PGRMCl neutralizing antibody (H-46; sc-98680, Santa Cruz Biotechnology, Santa Cruz, CA, USA). CCK-8 solution was added to each well, and the absorbance at $450 \mathrm{~nm}$ was measured using an Absorbance Microplate Reader (BioTek Instruments, Inc., Winooski, VT, USA).

Cell migration and invasion assays. Cell migration assay was performed using Transwell chambers ( $8 \mu \mathrm{m}, 24$-well insert; Corning Inc., Corning, NY, USA). The cells were added to the upper chamber with serum-free medium and the medium of the lower chamber contained 10\% FBS. Following incubation for $48 \mathrm{~h}$, the migrated cells in the lower chamber were stained with $0.1 \%$ crystal violet (C0121; Beyotime Institute of Biotechnology). Invasion assays were completed under the same conditions using Transwell membranes coated with Matrigel (BD Biosciences, Franklin Lakes, NJ, USA).

In vivo tumorigenesis and metastasis assays. The cells were collected and injected into the left ventricle of the hearts of 40 female athymic nude mice (SPF grade; 4-6 weeks old, weighing 18-22 g; Institute of Zoology, Chinese Academy of Sciences, Shanghai, China) subjected to oophorectomy using 


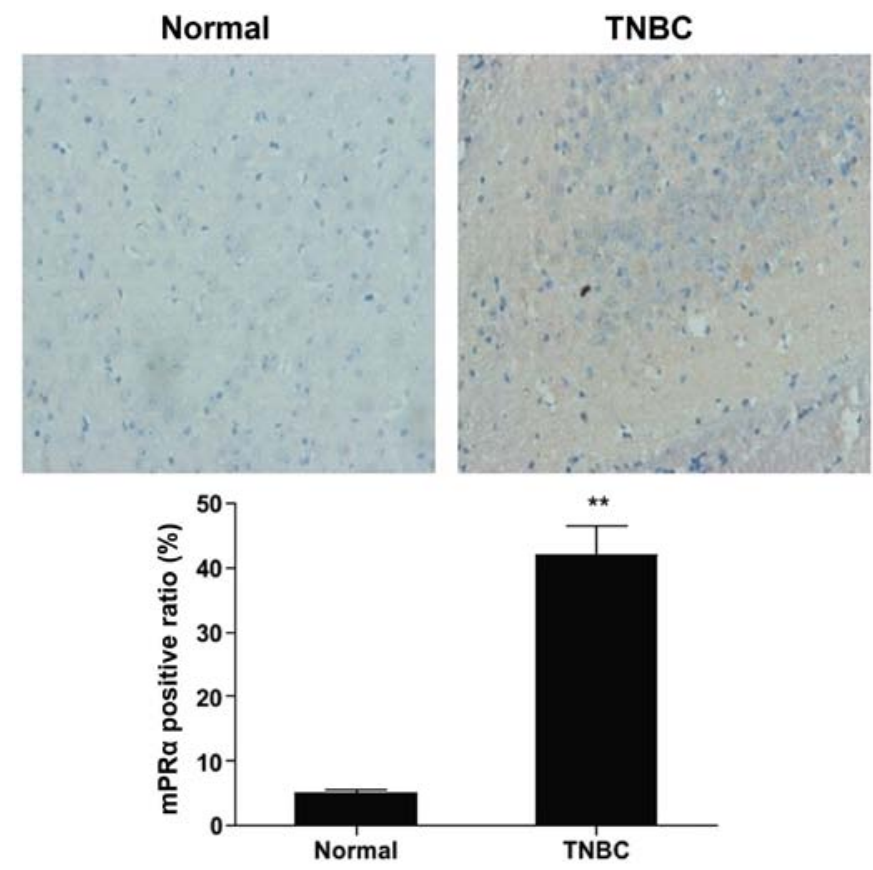

Figure 1. Membrane progesterone receptor $\alpha(\mathrm{mPR} \alpha)$ is overexpressed in triple-negative breast cancer (TNBC) tissues. The expression of $\mathrm{mPR} \alpha$ was examined by immunohistochemical staining in TNBC tissues and adjacent normal breast tissues. Magnification, $\mathrm{x} 200$. The $\mathrm{mPR} \alpha$-positive ratio of $\mathrm{mPR} \alpha$ in the TNBC tissues was significantly higher than that in the adjacent normal breast tissues $\left({ }^{* *} \mathrm{P}<0.01\right)$.

standard procedures. The mice were sacrificed after 5 weeks and a CT scan was performed for the analysis of brain metastasis formation. The brains were then collected, fixed and embedded in $4 \%$ paraformaldehyde. Terminal deoxynucleotidyltransferase-mediated dUTP nick-end labeling (TUNEL) assay was carried out following the manufacturer's instructions of the DeadEnd ${ }^{\mathrm{TM}}$ Colorimetric TUNEL system kit (Promega). All animal experiments were carried out following the approval of the Institutional Review Board of Shanghai Seventh People's Hospital; approval no. 2015011601)

Statistical analysis. Data analyses were carried out using SPSS v17.0 software with the Student's t-test. The results are presented as the means \pm SE. Statistical significance was determined at $\mathrm{P}<0.05$.

\section{Results}

$m P R \alpha$ is overexpressed in TNBC tissues and is associated with clinicopathlogical characteristics. To evaluate the expression of $\mathrm{mPR} \alpha$ in human TNBC tissues, we first determined the expression level of $\mathrm{mPR} \alpha$ by immunohistochemistry. Typical immunostaining of $\mathrm{mPR} \alpha$ in normal and TNBC specimens is shown in Fig. 1. The positive expression of $\operatorname{mPR} \alpha$ was mainly observed in the cytoplasm and/or cell membrane. In the normal breast tissues, mPR $\alpha$ was detected at low levels; the ductal and alveolar epithelial cells were shown to be negative or weakly positive and the myoepithelial cells were shown to be moderately positive for mPR $\alpha$. By contrast, all 55 TNBC tissues were stained moderately to strongly positive for mPR $\alpha$ antibody. The expression of $\mathrm{mPR} \alpha$ tended to decrease with
Table I. Correlation between the $\mathrm{mPR} \alpha$ expression level and clinicopathological parameters in the 55 patients with TNBC.

\begin{tabular}{lccc}
\hline & \multicolumn{2}{c}{ mPR $\alpha$ expression level } & \\
\cline { 2 - 3 } Characteristics & Moderate & Strong & P-value \\
\hline Age (years) & & & \\
$\geq 50$ & 12 & 13 & \\
$<50$ & 16 & 14 & $>0.05$ \\
Menopausal status & & & \\
Pre-menopause & 15 & 14 & $>0.05$ \\
Menopause & 14 & 12 & \\
Histological grade & & & $>0.05$ \\
Grade I & 11 & 7 & \\
Grade II & 8 & 10 & \\
Grade III & 9 & 10 & $<0.01$ \\
TNM stage & & & \\
I-II & 18 & 20 & \\
III-IV & 7 & 10 &
\end{tabular}

$\mathrm{mPR} \alpha$, membrane localized progestin receptor $\alpha$; TNBC, triple-negative breast cancer.

the increasing TNM stage $(\mathrm{P}<0.05)$, while no correlation was observed between mPR $\alpha$ expression and the patient age, menopausal state or histological grade (Table I).

Progesterone suppresses the growth of $m P R \alpha^{+}$human TNBC cells. To determine whether progesterone affects the growth of TNBC cells with a different $\mathrm{mPR} \alpha$ status, we first established stably transfected TNBC cell lines with different mPR $\alpha$ expression levels. The MB231 and MB231br cells are TNBC cells that do not express ER,PR and HER2; mPR $\alpha$ expression was detected at higher levels in the MB231br cells compared with the MB231 cells (Fig. 2A and B). Subsequently, mPR $\alpha$ fulllength expression vector or $\mathrm{mPR} \alpha$-shRNA were transfected into the MB231 and MB231br cells, respectively. The overexpression or knockdown of $\mathrm{mPR} \alpha$ in the MB231 or MB231br cells was confirmed by RT-qPCR (Fig. 2A) and western blot analysis (Fig. 2B). Cell proliferation was analyzed by CCK-8 assay in the presence of various concentrations of progesterone $(20 \mathrm{ng} / \mathrm{ml}$, $40 \mathrm{ng} / \mathrm{ml}$ and $80 \mathrm{ng} / \mathrm{ml}$ ). As shown in Fig. 2C, the proliferation of the MB231br (MB231br-shNC) and MB231-mPR $\alpha$ (MB231 cells transfected with $\mathrm{mPR} \alpha$ overexpression vector) cells, but not that of the MB231br-shmPR $\alpha$ (MB231br cells transfected with shRNA targeting $\mathrm{mPR} \alpha$ ) and MB231 (MB231-vector) cells was inhibited by progesterone treatment in a dose-dependent manner. We then analyzed cell apoptosis by examining the expression of cleaved caspase- 3 by western blot analysis following treatment with progesterone. As shown in Fig. 2D, the expression of cleaved caspase-3 was upregulated in both the MB231br and MB231-mPR $\alpha$ cells, but not in the MB231br-shmPR $\alpha$ or MB231 cells following treatment with progesterone. Thus, these results indicate that progesterone suppresses the growth of $\mathrm{mPR} \alpha^{+} \mathrm{TNBC}$ cells by inhibiting cell proliferation and inducing cell apoptosis. 

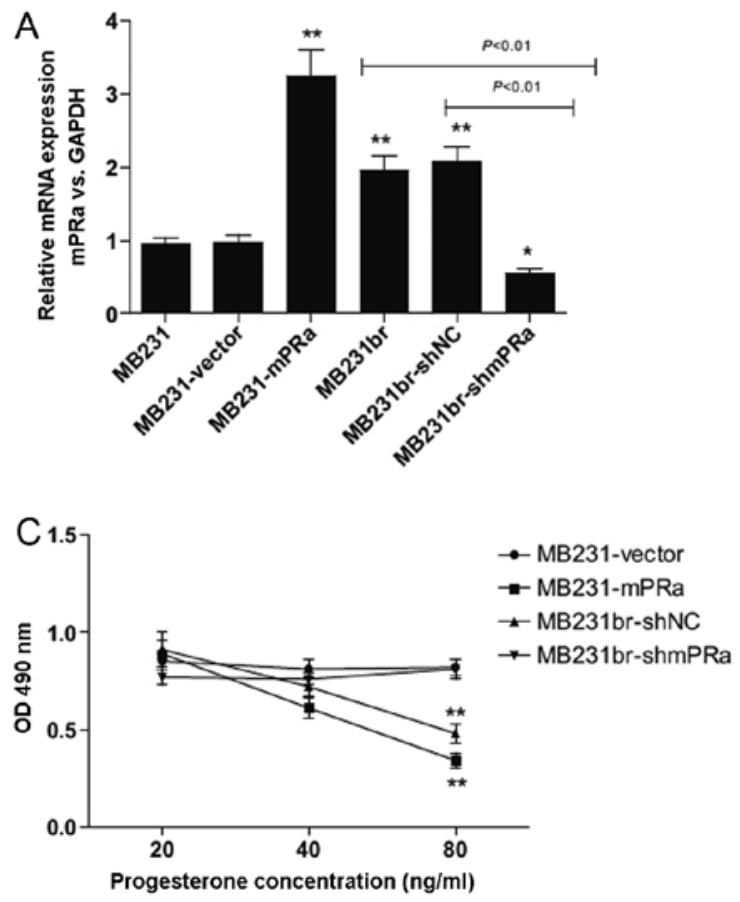
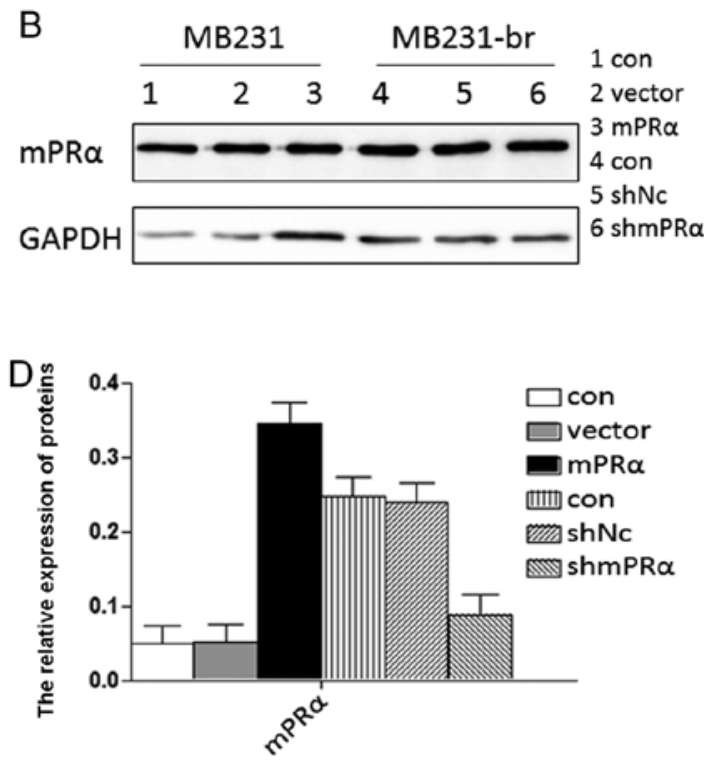

Figure 2. Progesterone suppresses the growth of membrane progesterone receptor $\alpha\left(\mathrm{mPR} \alpha^{+}\right)$human triple-negative breast cancer (TNBC) cells. (A) The mRNA expression levels of $\mathrm{mPR} \alpha$ in TNBC cells were detected by RT-qPCR following transfection with mPR $\alpha$ full-length expression vector or mPR $\alpha$-shRNA. " $\mathrm{P}<0.05$, ${ }^{* *} \mathrm{P}<0.01$. (B) The protein levels of $\mathrm{mPR} \alpha$ in breast cancer cells were analyzed by western blot analysis following transfection with $\mathrm{mPR} \alpha$ full-length expression vector or mPR $\alpha$-shRNA. GAPDH protein was used as the loading control. (C) Proliferation of TNBC cells with different mPR $\alpha$ expression status was evaluated by CCK- 8 assay following treatment with indicated concentrations of progesterone. Data are shown as the means \pm SD that represents as least 3 independent experiments. "P<0.05. (D) The expression of cleaved caspase-3 in TNBC cells with different mPR $\alpha$ expression status was analyzed by western blot analysis following treatment with $40 \mathrm{ng} / \mathrm{ml}$ progesterone for $48 \mathrm{~h}$. The $\mathrm{mPR} \alpha$-positive ratio of $\mathrm{mPR} \alpha$ in TNBC tissues was significantly higher than that in the adjacent normal breast tissues $\left({ }^{* *} \mathrm{P}<0.01\right)$.

Progesterone suppresses the migration and invasion of $m P R \alpha^{+}$human TNBC cells. We then examined the effects of progesterone on the migration and invasion of TNBC cells with a different $\mathrm{mPR} \alpha$ expression status. As shown in Fig. 3A, following treatment with progesterone, the MB231br cells (untreated group, 168 \pm 29 cells/field vs. treated group $95 \pm 12$ cells/field, $\mathrm{P}<0.05$ ), but not the MB231 cells (untreated group, $97 \pm 19$ cells/field vs.treated group $89 \pm 12$ cells/field) exhibited decreased migration. This inhibition was blocked by the knockdown of mPR $\alpha$ expression in the MB231br cells (untreated group, $166 \pm 25$ cells/field vs. treated MB231br-shmPR $\alpha$ group, $176 \pm 30$ cells/field) (Fig. 3A). Moreover, the introduction of exogenous mPR $\alpha$ cDNA into the MB231 cells enhanced the responsiveness of the cells to progesterone treatment, decreasing migration (untreated group, 103 \pm 22 cells/field vs. treated MB231-mPR $\alpha$ group 62 \pm 14 cells/field)(Fig.3A). Similarly, in the presence of progesterone, the MB231br cells (untreated group, $95 \pm 16$ cells/field vs. treated group $58 \pm 10$ cells/field, $\mathrm{P}<0.05$ ) and MB231-mPR $\alpha$ cells (untreated group, $74 \pm 14$ cells/field vs.treated group $42 \pm 9$ cells/field, $\mathrm{P}<0.05$ ), but not the MB231 (untreated group, $72 \pm 12$ cells/field vs. treated group $68 \pm 15$ cells/field) or MB231br-shmPR $\alpha$ cells (untreated group, 88 \pm 12 cells/field vs. treated group $93 \pm 9$ cells/field) exhibited a decreased invasion (Fig. 3B). Thus, these results indicate that progesterone suppresses the migration and invasion of $\mathrm{mPR} \alpha^{+} \mathrm{TNBC}$ cells.

The inhibitory effects of progesterone in $m P R P \alpha^{+}$human TNBC cells are not mediated by either PR or PGRMCl. Although the MB231br and MB231 cells are basically negative for nuclear
PR expression, it has been reported that cancer cells may repress PR in response to sex hormone treatments (20). In this study, in order to exclude the role of PR in the above-mentioned effects of progesterone on TNBC cells, the MB231br-shNC, MB231br-shmPR $\alpha$, MB231-vector and MB231-mPR $\alpha$ cells were co-incubated with progesterone plus RU486 (mifepristone), a PR-specific blocker. As expected, RU486 had no effects on the inhibitory effects of progesterone on cell proliferation, migration and invasion (Fig. 4A, B and C). PGRMCl is the other type of progesterone membrane receptor that mediates non-classical progestins actions (21). In this study, in order to exclude the possible role of PGRMCl in the inhibitory effects of progesterone on TNBC cells, the MB231br cells and MB231-mPR $\alpha$ cells were then co-incubated with progesterone plus PGRMCl neutralizing antibody. As shown in Fig. 4, PGRMCl neutralizing antibody had no effects on the inhibitory effects of progesterone on cell proliferation, migration and invasion. Thus, these results suggested that the inhibitory effects of progesterone on $\mathrm{mPR} \alpha^{+}$human TNBC cells are not mediated by either PR or PGRMCl.

Knockdown of mPRa expression impairs the inhibitory effects of progesterone on $m P R \alpha^{+}$tumor growth and metastasis in vivo. We further examined whether the knockdown of mPR $\alpha$ expression in TNBC cells can reverse the inhibitory effects of progesterone on tumor growth and metastasis in vivo. The MB231br-NC cells and MB231br-shmPR $\alpha$ cells were injected into the left ventricle of the hearts of athymic nude mice subjected to oophorectomy. Five weeks later, the 

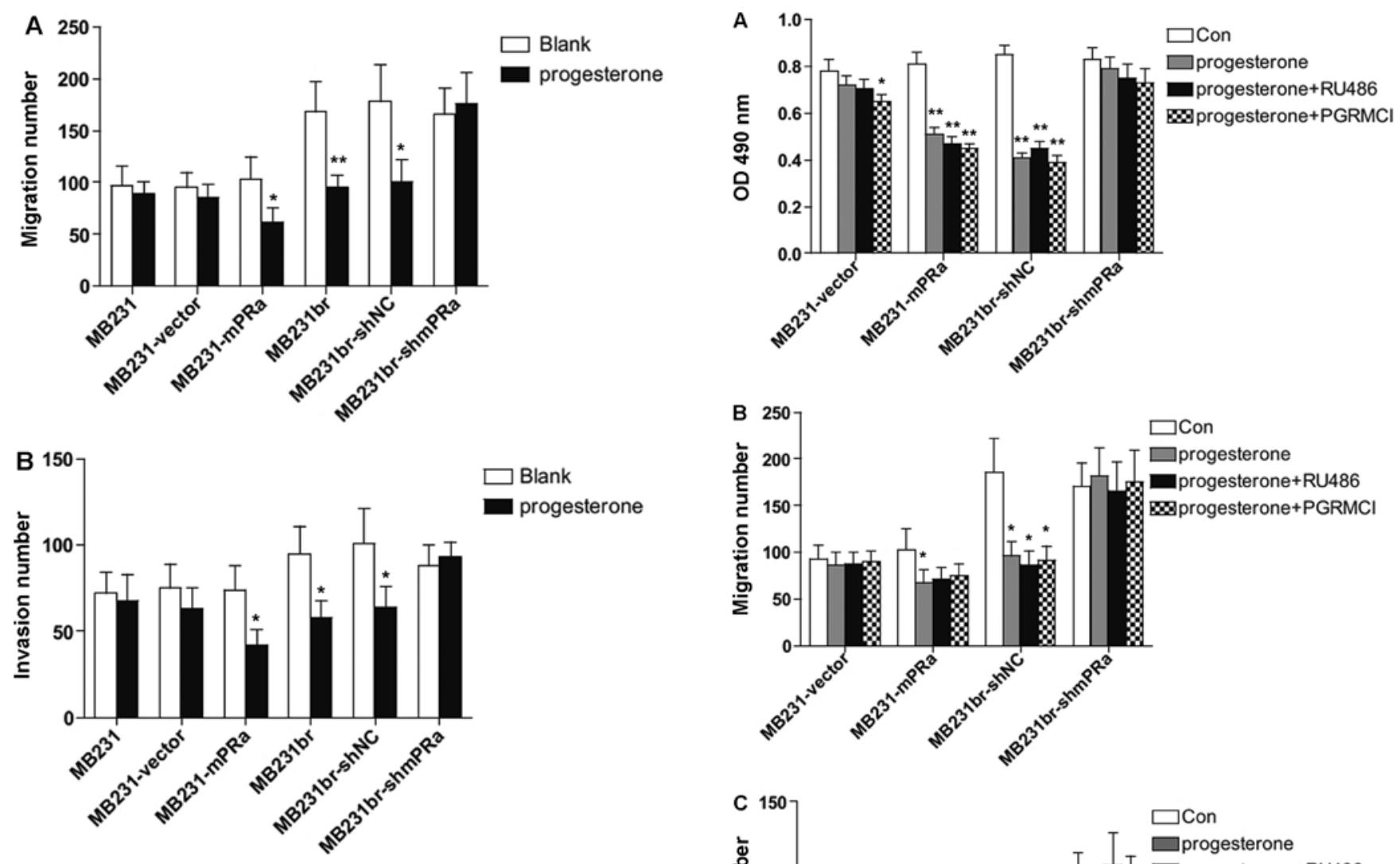

Figure 3. Progesterone suppresses migration and invasion of membrane progesterone receptor $\alpha\left(\mathrm{mPR} \alpha^{+}\right)$in human triple-negative breast cancer (TNBC) cells. (A) Transwell migration assay of the effects of progesterone on cell migration. (B) Transwell invasion assay of the effects of progesterone on cell migration. Data are shown as the mean \pm SD that represents as least 3 independent experiments. ${ }^{*} \mathrm{P}<0.05,{ }^{* *} \mathrm{P}<0.01$.

mice were euthanized, and H\&E staining and a CT scan were then performed for the analysis of brain metastasis formation. As shown in Fig. 5A and B, the MB231br-NC cells and MB231br-shmPR $\alpha$ cells formed brain metastases in the nude mice with oophorectomy. However, following treatment with progesterone, the MB231br-NC cells, but not the MB231brshmPR $\alpha$ cells, did not form brain metastases in the nude mice with oophorectomy. In addition, TUNEL assays of the brain tissues were performed. As shown in Fig. 5C, the MB231brshmPR $\alpha$ cells exhibited less tumor cell-positive staining and a significantly lower apoptotic index than the MB231br-NC cells $(\mathrm{P}<0.01)$. These results suggested that the knockdown of mPRa expression impairs the inhibitory effects of progesterone on $\mathrm{mPR} \alpha^{+}$tumor growth and metastasis in vivo.

\section{Discussion}

Progesterone plays an important role in mammary gland development in females and also appears to be involved in the development of breast cancer $(5,6)$. There is evidence to indicate that progesterone promotes rodent mammary carcinogenesis under certain conditions, in which PR is necessary for murine mammary gland tumorigenesis $(7,8)$. Breast cancer is a heterogeneous disease and several distinct subtypes exist, of which the triple-negative subtype has the worst clinical prognosis $(9,10)$. However, the role of progesterone as either

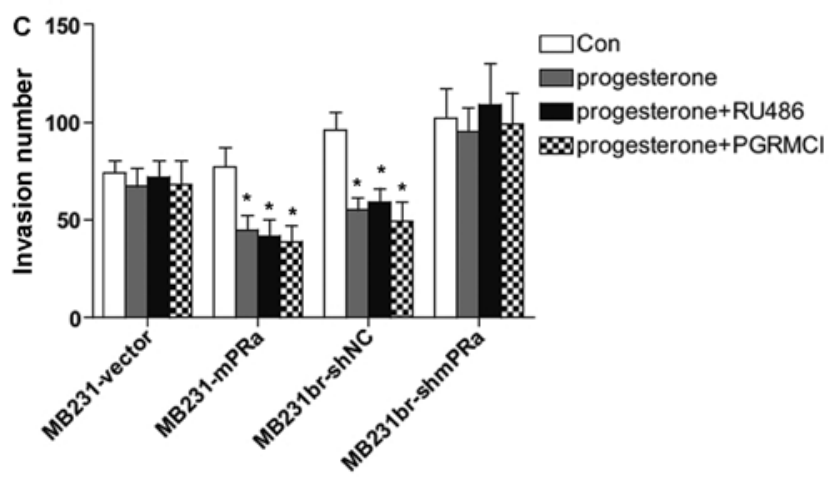

Figure 4. Inhibitory effects of progesterone on membrane progesterone receptor $\alpha\left(\mathrm{mPR} \alpha^{+}\right)$in human triple-negative breast cancer (TNBC) cells are not mediated by either PR or by PR membrane component 1 (PGRMCl). (A) Proliferation of MB231br and MB231-mPR $\alpha$ was evaluated by CCK-8 assay following treatment with progesterone plus RU486 (mifepristone) or PGRMCl neutralizing antibody. (B and C) Inhibitory effects of progesterone on the migration and invasion of MB231br and MB231-mPR $\alpha$. Data are the means \pm SD that represents as least 3 independent experiments. ${ }^{*} \mathrm{P}<0.05^{\text {*** }} \mathrm{P}<0.01$.

a promoter or inhibitor of TNBC that lacks the expression of PR has not yet been fully elucidated. In this study, we demonstrated that $\mathrm{mPR} \alpha$ was overexpressed in human TNBC tissues and the expression level of $\mathrm{mPR} \alpha$ was negatively associated with the TNM stage. We found that $\mathrm{mPR} \alpha$ mediates the inhibitory effects of progesterone on TNBC cell growth, migration and invasion in vitro, as well as growth and metastasis in vivo. These results provide evidence of a novel mechanism mediated by the progesterone-mPR $\alpha$ axis in the development and progression of TNBC.

The $\operatorname{mPR} \alpha$ receptor has been associated with manyphysiologic functions in vertebrates. It induces oocyte maturation, stimulates sperm hypermotility, modulates immune function, downregulates gonadotropin-releasing hormone $(\mathrm{GnRH})$ secretion and adjusts human myometrial 
A

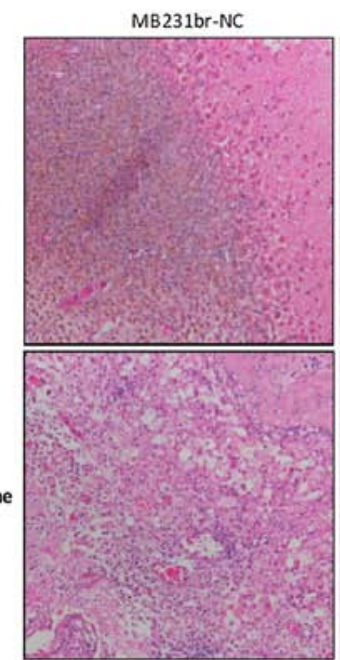

MB231br-NC

B

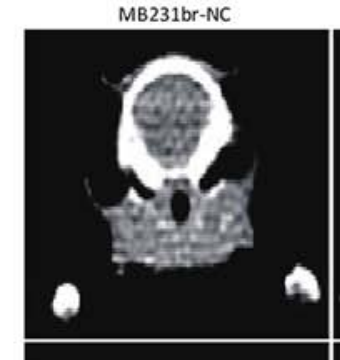

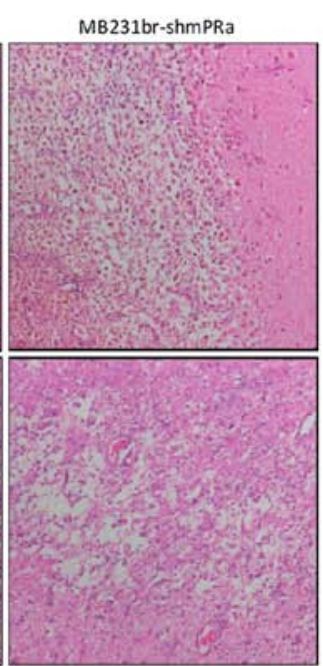

MB231br-shmPRa

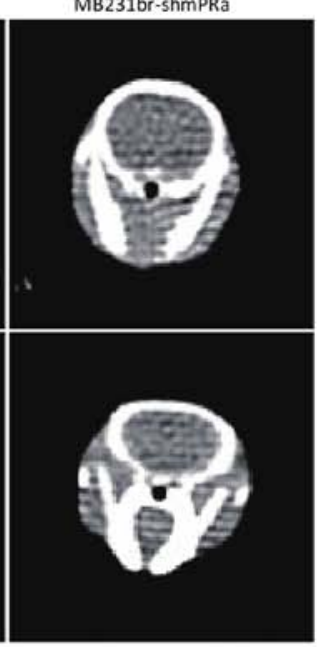

C
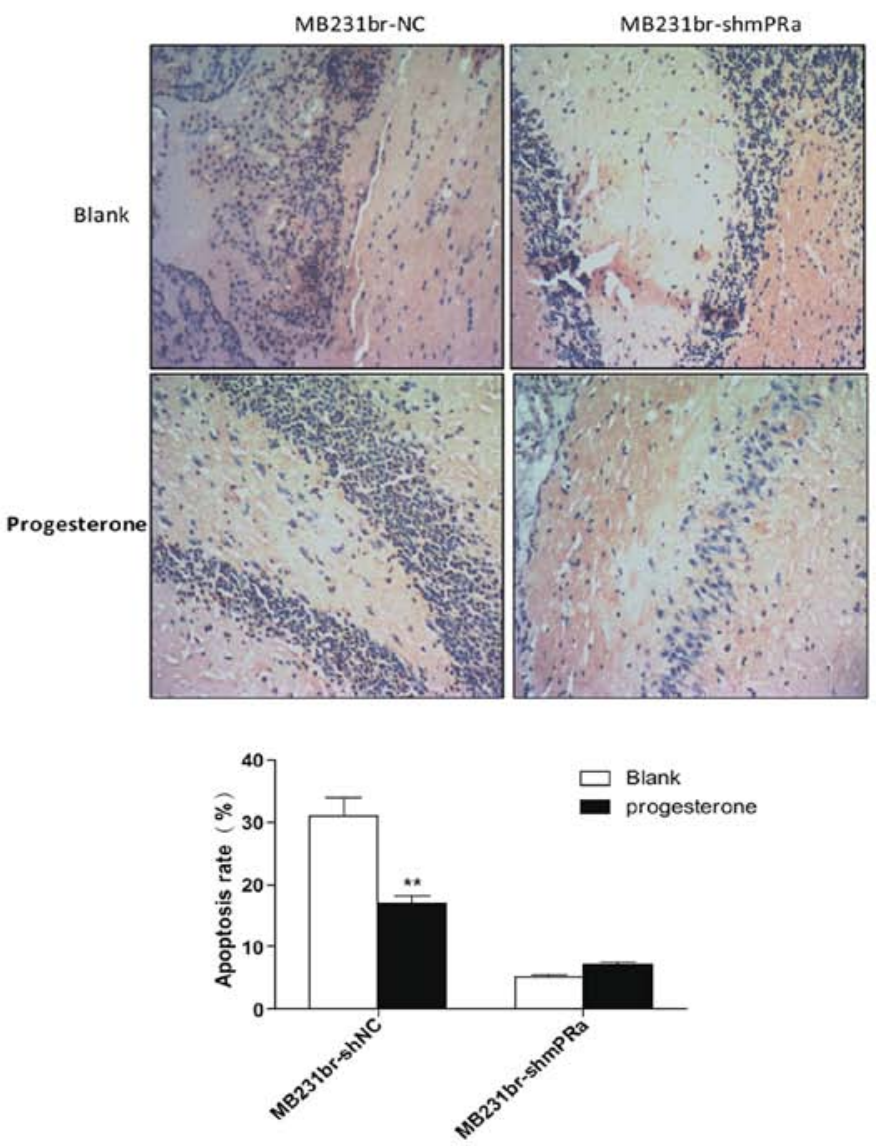

Figure 5. Knockdown of membrane progesterone receptor $\alpha(\mathrm{mPR} \alpha)$ expression impairs the inhibitory effects of progesterone on $\mathrm{mPR} \alpha^{+}$tumor growth and metastasis in vivo. (A) MB231br-NC cells and MB231br-shmPR $\alpha$ cells were injected into the left ventricle of the heart ofathymic nude mice with oophorectomy. Five weeks later, the mice were euthanized and H\&E staining was performed for the analysis of brain metastasis formation. (B) Five weeks later, mice were euthanized and a CT scan was performed for the analysis of brain metastasis formation. (C) TUNEL assays were performed in brain tissues from nude mice injected with MB231br-NC cells and MB231br-shmPR $\alpha$ cells and treated with progesterone. ${ }^{* *} \mathrm{P}<0.01$

cell contractility $(11,17,21-23) . \mathrm{mPR} \alpha$ was first identified in human breast cancer biopsies and epithelial-derived breast cancer cell lines by Dressing et al (18). Recently, it has been identified as an intermediary factor of the progestin-induced intracellular signaling cascades in $\mathrm{PR}^{-}$breast cancer cell lines in vitro $(15,18,19) . \mathrm{mPR} \alpha$ mediates epithelial-mesenchymal transition (EMT) through the activation of the PI3K/Akt pathway (19). In this study, the expression of mPR $\alpha$ was detected in both normal and malignant breast tissues and its expression level was negatively associated with the TNM stage of TNBC, which is consistent with previous results $(18,19,24)$. However, knowledge of the aberrant expression and potential role of mPR $\alpha$ in TNBC remains largely unknown. In this study, we demonstrated that progesterone suppressed the growth, migration and invasion of human TNBC cells via $\mathrm{MPR} \alpha$ in vitro and in vivo. Importantly, these effects induced by progesterone treatment were significantly blocked by transfection with mPR $\alpha$-specific shRNA. Therefore, $\operatorname{mPR} \alpha$ may contribute to cancer development, proliferation and metastasis in TNBC.
Classically, progesterone exerts its effects through the binding of nuclear PR and subsequently activates downstream pathways $(7,8)$. A previous study demonstrated that cancer progenitor cells may proliferate and express PR in response to sex hormone treatments (6); thus, the classical nuclear PR was first considered as a molecular mediator of the progesterone's inhibitory effects on TNBC cells even though they are basically negative for nuclear PR expression in normal culture condition. PGRMCl is the other type of progesterone membrane receptor that mediated the non-classical progestins actions (21). To exclude the possible role of PR or PGRMCl in the inhibitory effects of progesterone on TNBC cells, we introduced RU486 or PGRMCl neutralizing antibody into the culture system; neither of these had an effect on the inhibitory effects of progesterone. Thus, our data demonstrate that the status of $\mathrm{mPR} \alpha$ in TNBC cells play an essential role in determining the cell biological behavior of TNBC in responding to progesterone treatment. However, the detailed molecular mechanisms need to be further explored in the future. 
In conclusion, our study has provided experimental evidence indicating a role for progesterone in inhibiting TNBC development and progression. Although the detailed mechanisms require further investigation, a strong link between $\mathrm{mPR} \alpha$ expression and the effects of progesterone may provide a possible explanation as to how progesterone suppresses TNBC progression. Progesterone may play a duel role in breast cancer; therefore, a better understanding of the role of the progesterone-mPR $\alpha$ axis in various subtypes of breast cancer may help to provide insight into its complex function. In conclusion, our study indicates that progesterone suppresses TNBC cell growth and metastasis to the brain via $\mathrm{mPR} \alpha$, and may therefore serve as a potential target in the treatment of TNBC.

\section{Acknowledgements}

This study was supported by the Shanghai Natural Science Foun dation (grant no. 12ZR1422800), the Foundation of Shanghai TCM Oncology of TCM clinical key subject (ZYXK2012010) and the Foundation of Pudong New Area tumor key subject groups (PWZxq2014-12).

\section{References}

1. Foulkes WD, Smith IE and Reis-Filho JS: Triple-negative breast cancer. N Engl J Med 363: 1938-1948, 2010.

2. de Ruijter TC, Veeck J, de Hoon JP, van Engeland M and Tjan-Heijnen VC: Characteristics of triple-negative breast cancer. J Cancer Res Clin Oncol 137: 183-192, 2011.

3. Rakha EA and Chan S: Metastatic triple-negative breast cancer. Clin Oncol (R Coll Radiol) 23: 587-600, 2011.

4. Macias H and Hinck L: Mammary gland development. Wiley Interdiscip Rev Dev Biol 1: 533-557, 2012.

5. Kuhl H and Schneider HP: Progesterone - promoter or inhibitor of breast cancer. Climacteric 16 (Suppl 1): 54-68, 2013.

6. Axlund SD and Sartorius CA: Progesterone regulation of stem and progenitor cells in normal and malignant breast. Mol Cell Endocrinol 357: 71-79, 2012.

7. Lydon JP, Ge G, Kittrell FS, Medina D and O'Malley BW: Murine mammary gland carcinogenesis is critically dependent on progesterone receptor function. Cancer Res 59: 4276-4284, 1999.

8. Obr AE and Edwards DP: The biology of progesterone receptor in the normal mammary gland and in breast cancer. Mol Cell Endocrinol 357: 4-17, 2012 .

9. Yersal $\mathrm{O}$ and Barutca S: Biological subtypes of breast cancer: prognostic and therapeutic implications. World J Clin Oncol 5: 412-424, 2014.

10. Shah R, Rosso K and Nathanson SD: Pathogenesis, prevention, diagnosis and treatment of breast cancer. World J Clin Oncol 5: 283-298, 2014.

11. Zhu Y, Rice CD, Pang Y, Pace M and Thomas P: Cloning, expression, and characterization of a membrane progestin receptor and evidence it is an intermediary in meiotic maturation of fish oocytes. Proc Natl Acad Sci USA 100: 2231-2236, 2003.
12. Zhu Y, Bond $\mathbf{J}$ and Thomas P: Identification, classification, and partial characterization of genes in humans and other vertebrates homologous to a fish membrane progestin receptor. Proc Natl Acad Sci USA 100: 2237-2242, 2003.

13. Chapman NR, Kennelly MM, Harper KA, Europe-Finner GN and Robson SC: Examining the spatio-temporal expression of mRNA encoding the membrane-bound progesterone receptor-alpha isoform in human cervix and myometrium during pregnancy and labour. Mol Hum Reprod 12: 19-24, 2006.

14. Dosiou C, Hamilton AE, Pang Y, Overgaard MT, Tulac S, Dong J, Thomas P and Giudice LC: Expression of membrane progesterone receptors on human T lymphocytes and Jurkat cells and activation of G-proteins by progesterone. J Endocrinol 196: 67-77, 2008

15. Dressing GE and Thomas P: Identification of membrane progestin receptors in human breast cancer cell lines and biopsies and their potential involvement in breast cancer. Steroids 72: 111-116, 2007.

16. Fernandes MS, Pierron V, Michalovich D, Astle S, Thornton S, Peltoketo H, Lam EW, Gellersen B, Huhtaniemi I, Allen J, et al: Regulated expression of putative membrane progestin receptor homologues in human endometrium and gestational tissues. J Endocrinol 187: 89-101, 2005.

17. Karteris E, Zervou S, Pang Y, Dong J, Hillhouse EW, Randeva HS and Thomas P: Progesterone signaling in human myometrium through two novel membrane G protein-coupled receptors: potential role in functional progesterone withdrawal at term. Mol Endocrinol 20: 1519-1534, 2006.

18. Dressing GE, Alyea R, Pang Y and Thomas P: Membrane progesterone receptors (mPRs) mediate progestin induced antimorbidity in breast cancer cells and are expressed in human breast tumors. Horm Cancer 3: 101-112, 2012.

19. Zuo L, Li W and You S: Progesterone reverses the mesenchymal phenotypes of basal phenotype breast cancer cells via a membrane progesterone receptor mediated pathway. Breast Cancer Res 12: R34, 2010.

20. Dai D, Wolf DM, Litman ES, White MJ and Leslie KK: Progesterone inhibits human endometrial cancer cell growth and invasiveness: down-regulation of cellular adhesion molecules through progesterone B receptors. Cancer Res 62: 881-886, 2002.

21. Thomas P: Characteristics of membrane progestin receptor alpha (mPRalpha) and progesterone membrane receptor component 1 (PGMRC1) and their roles in mediating rapid progestin actions. Front Neuroendocrinol 29: 292-312, 2008.

22. Sleiter N, Pang Y, Park C, Horton TH, Dong J, Thomas P and Levine JE: Progesterone receptor A (PRA) and PRB-independent effects of progesterone on gonadotropin-releasing hormone release. Endocrinology 150: 3833-3844, 2009.

23. Tubbs $\mathrm{C}$ and Thomas P: Progestin signaling through an olfactory $\mathrm{G}$ protein and membrane progestin receptor-alpha in atlantic croaker sperm: potential role in induction of sperm hypermotility. Endocrinology 150: 473-484, 2009.

24. Xie M, Zhu X, Liu Z, Shrubsole M, Varma V, Mayer IA, Dai Q, Chen Q and You S: Membrane progesterone receptor alpha as a potential prognostic biomarker for breast cancer survival: a retrospective study. PLoS One 7: e35198, 2012. 